\title{
THE RADIO PROPERTIES OF SEYFERT GALAXIES IN THE 12-MICRON AND CFA SAMPLES
}

\author{
Brian Rush ${ }^{1}$ and Matthew A. Malkan \\ Department of Physics and Astronomy, University of California, Los Angeles, CA 90095-1562; \\ rush,malkan@astro.ucla.edu \\ and \\ Richard A. Edelson \\ Department of Physics and Astronomy, 203 Van Allen Hall, University of Iowa, Iowa City, IA 52242; \\ edelson@spacly.physics.uiowa.edu
}

\begin{abstract}
We report the results of 20,6 , and $2 \mathrm{~cm}$ VLA and $1.5 \mathrm{~cm}$ OVRO observations of two similar AGN samples: the optically-selected CfA Seyfert galaxies and the bolometricflux-limited $12 \mu \mathrm{m}$ active galaxy sample. Every object observed was detected at $6 \mathrm{~cm}$. Only $\sim 6-8 \%$ of the $12 \mu \mathrm{m}$-sample Seyferts (3-4 objects) are radio-loud (and none of the CfA sample), as compared to $15-20 \%$ for the BQS quasars. These radio-loud objects are compact and have flat spectra, distinguishing them from the more common radio-quiet objects.

The 6-20 $\mathrm{cm}$ slopes of the Seyfert $1 \mathrm{~s}$ and $2 \mathrm{~s}$ are similar, with average values of $\left\langle\alpha_{6 c m}^{20}\right\rangle=-0.66$ and -0.71 , respectively. Although several Seyfert 1s are significantly flatter than this in their $6-20$ and/or 1.5 - $6 \mathrm{~cm}$ slopes, there is no systematic trend for either Seyfert type to display upward or downward spectral curvature.

Excluding the radio-loud quasars, the integrated $6 \mathrm{~cm}$ radio luminosity is linearly proportional to the $60 \mu \mathrm{m}$ luminosity over several orders of magnitude, with on average twice the radio power of normal spirals of the same far-infrared power. About half of the objects show extended $6 \mathrm{~cm}$ emission, contributing on average $33 \%$ of the total flux. Thus the luminosities of these extended components alone are comparable to normal spirals of similar infrared luminosities.

The $12 \mu \mathrm{m}$ sample radio luminosity function is slightly higher than that of the CfA sample. The integrated space density of Seyfert $2 \mathrm{~s}$ is $\sim 2$ times that of Seyfert 1s over their common range in luminosity. In terms of the standard unified model, this ratio in space density corresponds to a typical half-angle of the torus of $\theta \sim 48^{\circ}$.
\end{abstract}

Subject headings: galaxies: active — infrared: galaxies: — galaxies: Seyfert — radio continuum: galaxies - surveys

\footnotetext{
${ }^{1}$ Current Address: The Carnegie Observatories, 813 Santa Barbara St., Pasadena, CA 91101-1292
} 


\section{Introduction}

The most fundamental division between different types of extragalactic radio sources is radio-loud vs. radio-quiet, where radio-loud objects are those with much higher radio luminosities, both in absolute terms $\left(L_{6 \mathrm{~cm}}>10^{42} \mathrm{erg} \mathrm{s}^{-1}\right.$-Miller, Peacock, \& Mead 1990), and relative to other wavelengths (e.g., a factor of $\sim 10^{4}$ more luminous at $6 \mathrm{~cm}$ for a given [O III] luminosity-Wilson \& Colbert 1995). Radioquiet active galaxies and quasars (generically referred to as AGN herein) can further be divided into radioquiet quasars, Seyfert 1s, and Seyfert 2s (the difference between the first two being, perhaps, just total absolute luminosity).

Furthermore, quantifying any differences between the average radio properties of various types (e.g. radio-loud vs. radio-quiet objects or Seyfert 1s vs. Seyfert 2s) also has direct applications towards unified models which relate different classes through effects such as relativistic beaming, or orientationdependent obscuration. According to the basic unified model for Seyfert galaxies, the nuclei of Seyfert $2 \mathrm{~s}$ are intrinsically similar to those of Seyfert 1s, yet viewed edge-on, so that our view to the inner-most parts of the nucleus, including the "broad-line" region, is obscured by a molecular torus. More complicated models expand on this picture by including other parameters which vary from object to object, such as the thickness of the torus or mass of the central engine. These models can be tested observationally by the fact that they predict many differences between the multiwavelength properties of type 1 and type 2 Seyfert galaxies (Antonucci 1993). A general prediction of this model is that isotropic properties (originating at radii outside of the torus) will be similar in Seyfert 1s and 2s, but that emission from the innermost regions will be orientation-dependent and thus will differ between Seyfert 1s and 2s.

As an example, a potential challenge to the simplest form of these unified models was found in early studies concluding that type 2 Seyferts have stronger and larger nuclear radio sources than type 1 Seyferts (de Bruyn \& Wilson 1978; Meurs \& Wilson 1984; Ulvestad \& Wilson 1984a,b). However, these studies were influenced by selection effects in the Markarian sample, causing the weaker Seyfert 2 galaxies to be omitted from the samples. In contrast, samples selected largely from the CfA redshift survey show no significant difference between the radio sources in the different Seyfert types (Edelson 1987-hereafter E87; Ulvestad \& Wilson 1989; Giuricin et al. 1990). Determining which result holds for the true population of Seyferts in the local universe requires observations of a well-defined sample large enough for significant statistical analysis.

We have therefore obtained 6 and $20 \mathrm{~cm}$ data from the VLA (and some single-dish $1.5 \mathrm{~cm}$ fluxes) for two samples of bright, nearby AGN which are mostly radio-quiet, specifically classified as Seyfert 1s and Seyfert 2s. We use these data to investigate the characteristics of the observed radio and multiwavelength properties of Seyferts and to study the differences in these properties between Seyfert classes.

Since radio-quiet AGN reside in host galaxies that may also contribute significantly to the overall observed spectral energy distribution, we also need to determine the relative contribution from the central and extended components. With this in mind, we will discuss several of the results presented herein in terms of a two component model, with the central component (i.e., the nucleus plus other unresolved flux) accounts for most of the radio flux, but where an extended component, with a lower radio-infrared flux ratio, also contributes significantly.

In $\S 2$ (and Appendix A) we discuss the target selection and observations. The analysis is in $\S 3$; in $\S 3$ we discus radio spectral properties; in $\S$ compactness and extended emission; in $\S$ 国 the correlation between radio and infrared luminosities; in $\S 6$ the frequency of radio-loud objects in the $12 \mu \mathrm{m}$ sample; and in $\S$ 7 radio luminosity functions. A summary is given in $\S 8$

\section{Observations and Data Reduction}

The CfA and $12 \mu \mathrm{m}$ Seyfert galaxy samples are believed to be relatively free of selection effects and systematic biases that have plagued other samples such as the Markarian Seyferts (see Appendix A; also Huchra \& Burg 1992, RMS93). As the CfA sample is optically selected, while the $12 \mu \mathrm{m}$ sample was selected in the infrared, they will enable us to compare the radio properties of samples with very different selection criteria (mid-IR and optical, respectively), as well as to compare the properties of each sample to those of radio-selected objects. We therefore obtained VLA 6 and $20 \mathrm{~cm}$ data of virtually all objects in both samples which are observable from the VLA (with the exception of a few objects which were added 
to the final definition of these samples after we began this project).

The new observations presented in this paper were carried out during 1990-1991. Seven CfA Seyferts with missing VLA data or upper limits in E87 were observed during January 1990. The $12 \mu \mathrm{m}$-sample Seyferts (that were not also observed as part of the CfA sample in E87) were observed during two runs in March 1991. Single snapshots were taken with integration times of 10 minutes at $6 \mathrm{~cm}$ and 2.5 minutes at $20 \mathrm{~cm}$, for 34 objects in each band. Every object observed was detected above the $3 \sigma$ noise levels of 0.35 at $6 \mathrm{~cm}$, and most above $1.0 \mathrm{mJy}$ at $20 \mathrm{~cm}$. The observations presented in E87 (both the VLA and OVRO data), and also discussed here, were taken during July 1983.

To make comparisons at different wavelengths, we combined untapered D-array measurements at $20 \mathrm{~cm}$, tapered beam data at $6 \mathrm{~cm}$, and single-dish $1.5 \mathrm{~cm}$ observations, to achieve a fairly uniform beamwidth of $\sim 1^{\prime} .5$ FWHM. In addition, untapered (typical beam FWHM $\left.\sim 15^{\prime \prime}\right) 6 \mathrm{~cm}$ measurements are also used for measuring the flux of the compact region. Since the radio sources in nearby Seyferts are often partially resolved on longer baselines, this large beam will yield the most uniform database, without introducing biases by mixing data from different arrays. The VLA maps were calibrated with the standard AIPS software, CLEANed with the AIPS task MX, and finally we used the AIPS task IMFIT to fit to an elliptical Gaussian to each map to measure the central flux density. The reduction of the OVRO data is described in E87.

Potential variability is not likely to affect our results in any significant way, since most of our observations are at least quasi-simultaneous. The data at $6 \mathrm{~cm}$ and $20 \mathrm{~cm}$ (and, where it exists, at $2 \mathrm{~cm}$ ) were taken on the same day for any given object, and the OVRO $1.5 \mathrm{~cm}$ observations were made within two weeks of the VLA observations for the same object (except for NGC 5273, for which the $20 \mathrm{~cm}$ data were taken in 1991 and 1.5 and $6 \mathrm{~cm}$ data were taken in 1983).

Table 1 presents the data. Column 1 gives the name of each object, column 2 the Seyfert type, column 3 the sample (12 $\mu \mathrm{m}$ and/or CfA), and column 4 the redshift. The next columns gives the flux densities and uncertainties (in mJy) at $20 \mathrm{~cm}, 6 \mathrm{~cm}$ (both high (h) and low (l) resolution, for untapered and tapered beams, respectively), and $1.5 \mathrm{~cm}$. (For the five objects noted in the table footnote, this last data point is actually a $2 \mathrm{~cm}$ observation taken with the VLA and should be considered only as a lower limit, since the VLA beam size is much smaller than that at OVRO.) The quoted uncertainty is the quadratic sum of the statistical errors and an estimated $5 \%$ uncertainty in the calibration. Upper limits are at $3 \sigma$. The final column indicates where these data were first reported $(\mathrm{R}=$ this work; $\mathrm{E}=\mathrm{E} 87)$. We list all of our new observed fluxes and those from E87 in Table 1, so that readers can readily have available the complete VLA data for both the $12 \mu \mathrm{m}$ and CfA samples.

In Table 2 we present derived properties. The first 3 columns are the same as in Table 1 . Columns 4 and 5 give the radio spectral indices, $\alpha_{1.5}^{6}$ and $\alpha_{6}{ }^{20}$, using the tapered $6 \mathrm{~cm}$ beam for accurate comparison to the other wavelengths. Columns 6 and 7 present the radio-IR spectral indices between $6 \mathrm{~cm}$ and both $60 \mu \mathrm{m}$ and $12 \mu \mathrm{m}$. The IRAS data were obtained from Rush, Malkan, \& Spinoglio (1993hereafter RMS93) for objects in the $12 \mu \mathrm{m}$ sample and from Edelson, Malkan, \& Rieke (1987) for those CfAsample Seyferts not in the $12 \mu \mathrm{m}$ sample. Column 8 gives the IRAS 25-60 $\mu \mathrm{m}$ spectral index. Columns 9 , 10 , and 11 give the $6 \mathrm{~cm}, 20 \mathrm{~cm}$, and $60 \mu \mathrm{m}$ monochromatic luminosities $\left(\nu L_{\nu}\right.$, in units of $\left.\mathrm{erg} \mathrm{s}^{-1}\right)$. Column 12 gives the radio-compactness parameter, defined as $R=S_{6 \mathrm{~cm}, h} / S_{6 \mathrm{~cm}, l}$, following E87. We stress that this $R$ parameter is not a ratio of the flux from the unresolved AGN nucleus to that from the entire galaxy, as others have used. Rather, it is a ratio of the "central" flux to that from the entire galaxy, where by "central" we mean the less-extended flux in a general sense. This central flux is not to be confused with the unresolved "nuclear" flux, since it would include this flux plus any double, triple, and/or jet-like sources associated with the nucleus, as well as circumnuclear or inner disk emission related to a starburst. With this in mind, we will refer to the numerator and denominator of this $R$ parameter (i.e., the $S_{6 \mathrm{~cm}, h}$ and $S_{6 \mathrm{~cm}, l}$ values) as the "central" and "total" $6 \mathrm{~cm}$ flux (where total $=$ central plus "extended") throughout this paper. The usefulness of this $R$ parameter as we have defined it is very similar to, but less powerful than a ratio of nuclear-to-extended flux. A plot of $R$ versus nuclear-to-extended flux for a sample of Seyferts flux should be monitonically increasing in general, with

\footnotetext{
${ }^{2}$ We assume a value of $\mathrm{H}_{0}=75 \mathrm{~km} \mathrm{~s}^{-1} \mathrm{Mpc}^{-1}$ throughout this paper.
} 
both ratios being larger in more nuclear-dominated sources. Thus, $R$ gives some indication of the extent to which an object is nuclear-dominated, i.e. how "compact" it is, hence the name "compactness parameter."

\section{Radio Spectral Properties}

We have measured the $6-20 \mathrm{~cm}$ spectral slope and, when possible, the $1.5-6 \mathrm{~cm}$ slope. The average value of $\alpha_{6}{ }^{20}$ is $-0.66 \pm 0.04$ for Seyfert 1 s and $-0.71 \pm 0.04$ for Seyfert 2s (all uncertainties quoted herein represent one standard deviation of the mean unless otherwise noted). For those objects with 1.5 or $2 \mathrm{~cm}$ observations, we have plotted $\alpha_{6}{ }^{20}$ versus $\alpha_{1.5}^{6}$ in Figure 1. (All spectral slopes referred to here are such that $S_{\nu} \propto \nu^{\alpha}$.) Symbols are explained in the caption. For the few objects for which we have $2 \mathrm{~cm} \mathrm{D-array}$ data instead of $1.5 \mathrm{~cm}$ OVRO single-dish data, we have assumed $\alpha_{1.5}^{6}=\alpha_{2}^{6} 3^{3}$. This figure is the same as Figure 3 in E87, with the addition of the points at $2 \mathrm{~cm}$, with the upper/lower limits displayed, and with changes in some object types due to better, more recent optical spectra.

We note that one tentative result suggested in E87 cannot be confirmed with this data, namely that Seyfert $1 \mathrm{~s}$ are more likely than Seyfert $2 \mathrm{~s}$ to have a high-frequency excess, i.e. spectral curvature. This can be seen by noting the solid line, which represents $\alpha_{1.5}^{6}=\alpha_{6}^{20}$. Most objects (for which both slopes are detections) are within $0.1-0.2$ of this line. Although this distance is larger than the typical intrinsic uncertainty in our measured slopes, it is on the order of, or smaller than, the typical uncertainties of the slope measurements which result from measuring the flux from less than the entire galaxy (and even from different regions at each wavelength). This point is illustrated in Figure 2, where we the compare our $6-20 \mathrm{~cm}$ (VLA) slopes with those measured for the whole galaxy from the single-dish fluxes in the northern sky survey (Becker, White, \& Edwards 1991; White \& Becker 1992; shown for all objects in both samples). As can be seen, the differences in slope

\footnotetext{
${ }^{3}$ These objects usually have very low fluxes at $2 \mathrm{~cm}$, indicating less high-frequency flux than the other galaxies in this plot. This is most likely due to the fact that the VLA in D-array has the greatest resolution at the shorter wavelengths and has resolved these objects, whereas the OVRO single-dish flux is from the entire galaxy. Thus, the $2 \mathrm{~cm}$ point is considered a lower limit, and thus the points in the plot are marked as lower limits to $\alpha_{1.5}^{6}$ (right-pointing arrows)
}

are typically $0.1-0.3$. Such differences would probably not cause any systematic change in Figure 1 if wholegalaxy fluxes were used, as is implied by the absence of any correlation in this plot between the slope difference and our slopes. Neither is there any trend with redshift, as one might expect if there is a strong radio color gradient in these galaxies, which could cause the slope difference to be stronger in nearby sources. (A similar result, of the same or smaller magnitude, is found when we compare our $1.5-6 \mathrm{~cm}$ slopes with those formed by combining our $1.5 \mathrm{~cm}$ single-dish fluxes with the Becker et al. $6 \mathrm{~cm}$ fluxes.)

Finally, we point out that there is one systematic effect apparent in Figure 1, namely that those few objects with at least one or both slopes being very flat are all Seyfert 1s. However, this does not comment on spectral curvature, because either slope can be the flat one. The dotted lines in Figure 1 enclose those objects in the lower left with both slopes steeper than -0.6 . All 8 objects outside of this box with detections in both axes are Seyfert 1s. The three objects with one or the other slope being very flat (i.e., outside of the box by twice the typical uncertainty discussed above), which would not simply result from measurement differences, are individually labeled. That the other 5 flat objects are all Seyfert 1s is probably also physically meaningful, since it is not likely that this would happen by chance, and since there is no general tendency (as would be seen in Figure 2) for Seyfert 1s to be flatter in our measurements than in the singledish measurements.

\section{Extended Emission}

To investigate the relation between the radiocompactness parameter, $R$, and other radio properties of Seyferts, we have plotted $\mathrm{L}_{6 \mathrm{~cm}}$ versus $R$ in Figure 3 . Here we see that the few radio-loudest objects are very compact, all having $R \sim 1$ ( $R$ is typically accurate to $\pm 10 \%$ - less for the fainter objects - hence the few values of $R$ greater than 1$)$. We note that the more luminous and compact objects in our sample are also among those with the flattest spectral slopes. These facts are consistent with models in which the radio-loud objects are compact, flat spectrum radio sources and that they have type-1 Seyfert nuclei with the radio emission directed towards our line of sight. The most luminous Seyfert 2s, on the other hand, have steeper spectra, but are still compact at our Darray resolution (they may, however, be shown to have 
less compact cores if observed with higher resolution). Three Seyfert $1 \mathrm{~s}$ and no Seyfert $2 \mathrm{~s}$ have $L_{6 \mathrm{~cm}}>10^{40}$, have the flattest spectra $\left(\alpha_{6}{ }^{20}>-0.2\right)$ and are very compact $(R=1)$.

Thus, we see a clear distinction in properties $\left(L_{6 \mathrm{~cm}}\right.$, $\alpha_{6}{ }^{20}$, and $R$ ) between the very few radio-loud objects and the more numerous, relatively radio-weak objects in our sample (see $\S$ f for further discussion of radio-loud versus radio-quiet objects in our sample). For the majority of the (radio-weak) galaxies, a slight trend in the same sense is found, with much scatter, indicating that some of these objects may be harboring very weak compact cores. This is shown by the straight line in Figure 3, which is the best-fit line to all objects in the plot (with slope $=2.41$ and $\mathrm{r}=0.23$ ), and the dashed line which excludes the 7 most luminous (labeled) galaxies (with slope $=1.56$ and $\mathrm{r}=0.17$; excluding only the two strictly radioloud objects would make the slope even steeper). (See below for explanation of the curved lines in this figure.)

We find that, although $\mathrm{L}_{6 \mathrm{~cm}}$ is correlated with distance in our sample (as expected; $\mathrm{r}=0.68$ ), $R$ is not $(\mathrm{r}=0.06)$, implying that the correlation between $\mathrm{L}_{6 \mathrm{~cm}}$ and $R$ is not simply an artifact of redshift. This also implies that the variation in $R$ in our sample represents the intrinsic range of extended $6 \mathrm{~cm}$ emission among Seyfert galaxies. Thus, we find in both samples that both Seyfert 1s and 2s have steep radio spectra with resolved structure on the $\gtrsim 1$ 'scale. This suggests that the low frequency and low resolution emission may be dominated by optically thin synchrotron emission from an optically thin source, such as the galactic disk.

The average value of $R$ for all galaxies is $\sim 0.83$, with no difference between Seyfert type or between the two samples. Roughly half of the objects have $\mathrm{R}<0.9$, i.e. extended $6 \mathrm{~cm}$ emission is found in about half of the observed galaxies, with an average value for $R$ among those objects of $\sim 0.67$ (i.e., an extended component contributes about $0.33 \% \pm 0.17 \%$ to the total flux). This represents a significant contribution from the underlying galaxy, which must be taken into account when considering measurements such as total luminosities where both the central and extended components are significant, as well as spectral slopes and flux ratios where the two combined components may have different values.

Accordingly, we have also plotted in Figure 3 several curved lines representing a simple physical model.
In this model, the total $6 \mathrm{~cm}$ luminosity is the sum of the central and extended components $\left(\mathrm{L}_{c e n}\right.$ and $\mathrm{L}_{\text {ext }}$, respectively), and $R$ is the ratio of central to total (i.e., central to central-plus-extended) luminosity. (Note that the extended component is a lower limit to the luminosity of the disk of the galaxy, as the latter will also emit at least some flux at radii within the "central" component.) Each curve starts at a given value of $\log L_{e x t}(36.5,37.5$, and 38.5 for the lower, middle, and upper curves, respectively) at $R=0$, and increases with $R$ (i.e., as the fraction of central luminosity increases). Although the scatter of the data is quite large, the general shape of these curves matches the data: luminosity is slightly correlated with compactness for small values of $R$, but increases sharply at the highest values of $R$. The curve representing an extended-component luminosity of $\log L_{\text {ext }}=37.5$ goes right through the center of the data (and roughly also the best-fit lines), but values an order of magnitude higher or lower than this are required to reproduce all the points.

\section{The Radio-Infrared Correlation}

Figure 4 shows a plot of the $6 \mathrm{~cm}$ versus $60 \mu \mathrm{m}$ monochromatic luminosities. The results of a bivariate regression to all the data (excluding the superluminal quasar 3C 120 and the BL Lac object OJ 287discussed further in $\S\left[\right.$ is $L_{6 \mathrm{~cm}} \propto L_{60 \mu \mathrm{m}}^{1.05}$, indistinguishable from a linear correlation. We therefore show, with dotted line \#1 the best fit obtained when the slope is constrained to 1 , yielding the relation $L_{6 \mathrm{~cm}}=10^{-b} \times L_{60 \mu \mathrm{m}}^{1}$, with $b=5.31$. The linear proportionality for normal galaxies (determined by Bicay \& Helou 1990, using their $20 \mathrm{~cm}-60 \mu \mathrm{m}$ relation and assuming a value of $\alpha_{6}{ }^{20}=-0.7$ ), which has the value $b=5.64$, is shown with line \#2. For comparison, we also show the lines from RMS93, representing their fit to Seyferts $(\# 3 ; b=5.0)$ and non-Seyferts $(\# 4$; $b=5.61$ ). Those fits were done using "survival analysis" procedures with the ASURV software package (La Valley, Isobe, \& Feigelson 1992) to account for many upper limits in the radio fluxes, particularly of Seyferts. (Such procedures assume that both the detected and undetected objects were drawn from the same homogeneous sample, which can explain why the fit to Seyferts from RMS93 is higher than that of this work.)

Comparing these lines shows Seyfert galaxies to have excess $6 \mathrm{~cm}$ emission relative to that at $60 \mu \mathrm{m}$, 
as compared to non-Seyferts, by about a factor of two. There is no significant difference in this relation between Seyfert 1s and 2s. This can be explained if Seyferts are not like normal spirals, but include a mix of "central" radio-plus-IR light with galaxy radio-plus-IR light. In this scenario, the central component, more dominated by the Seyfert nucleus is the component with the higher radio-IR flux ratio. To explain this, we have calculated curves similar to those in Figure 3. As in that case, we model the luminosity at $6 \mathrm{~cm}$ as the sum of central and extended components, and now we do the same for the $60 \mu \mathrm{m}$ luminosity. for the extended component, we use the value derived for normal galaxies by Bicay \& Helou (1990; $b=5.64$, as mentioned above). The different model curves represent the following variations of parameters: each of two sets of model curves starts at a locus (denoted by an open star), which corresponds to a given value of the extended-component luminosity at $6 \mathrm{~cm}\left(L_{\text {ext }}=10^{36.5}\right.$, and $10^{38.0}$, for the lower left and upper right sets, respectively). Four curves then span out from each star, corresponding to different color central components $(b=5.64,5.31,5.0$, and 4.5, representing normal galaxy-like, Seyfert-like, radiostrong, and very radio-strong colors; as labeled on the lower set of curves). Finally, along each of these curves, $R$ varies from 0.01 at the star up to 0.999 at the end of the curve (with transverse lines drawn at $R=0.50$ and $R=0.90$ ).

As defined, when $b_{c e n}=b_{e x t}=5.64$, the curves follow the line from Bicay \& Helou (1990) for normal galaxies, while when $b_{c e n}=5.31,5.0$, or 4.5 , the curves start at the normal galaxy line (at $R=0$ ) and asymptotically approach the Seyfert lines (as $R \rightarrow 1$ ). We see that the different sets of curves are highly degenerate. For example, a very compact object with $L_{\text {ext }} \sim 10^{36.5}$ may have a similar total luminosity to a less compact object with $L_{\text {ext }} \sim 10^{38}$. Furthermore, the fact that the scatter is much larger than the observational uncertainties in the data indicates that, some objects would have to have much higher or lower $6 \mathrm{~cm}-60 \mu \mathrm{m}$ flux ratios than the values used

\footnotetext{
${ }^{4}$ We stress that this is a simplified model and that the actual case is probably more complicated (for example, the radio-infrared ratio of the central component may increase with luminosity). However, such modeling will help to illustrate the extent to which the emission from Seyfert galaxies can be explained as resulting from the sum of a central plus an extended component, each with different values of the ratio of radio to infrared luminosity.
}

in this simplified model.

We apply this model further in Figure 5, where we plot the $25-60 \mu \mathrm{m}$ infrared slope versus the radio compactness parameter, $R$. We chose this infrared slope because it is often used to select for "warm" IRAS galaxies which are often Seyferts (e.g., Low et al. 1988). Here we also assume the constant $6 \mathrm{~cm}-$ $60 \mu \mathrm{m}$ ratio from Bicay \& Helou for the extended component. The different curves represent various values of this ratio for the central component. The highest (solid) curve assumes $b_{c e n}=b_{e x t}=5.64$, the next highest one $b_{c e n}=5.31$, and the two dotted ones $b_{c e n}=5.0$ and 4.5 , respectively. The two horizontal lines represent the values of the $25-60 \mu \mathrm{m}$ ratio assumed for the extended and central commponents (typical values for normal galaxies and for quasars in our $12 \mu \mathrm{m}$ sample, respectively). The curves all connect the lower horizontal line at $R=0$ to the upper horizontal line at $R=1$ (i.e., the values chosen for $\alpha_{25}^{60}$ simply determine the start and end points of each curve, without affecting their shape). These curves show us that a very radio-strong central component is necessary to match some of the data points given this model, while a range in the radio - infrared ratio of the central component is still necessary to match all the data. Alternatively, a cooler far-IR slope of the central component (i.e., still significantly hotter than the extended component, but not by as much as assumed in the model plotted), again combined with a wide range in radio strength, could also produce a family of curves which span most of the data points without requiring such extreme high values of radio strength (i.e., $b \sim 5$ instead of $b \sim 4.5$ would be sufficient).

\section{Radio-Loud Objects in the 12-Micron Sam- ple}

From these figures we see that a few objects in the $12 \mu \mathrm{m}$ sample can clearly be distinguished from the rest as being radio-loud. For comparison, Kellerman et al. (1989) observed the BQS quasars at $6 \mathrm{~cm}$, finding about $15-20 \%$ of the that sample to be radioloud, having $\log F_{\nu, \text { rad }} / F_{\nu, \text { opt }}$ from 1.5 to 3 , while the rest have values around -1 to 1.5 . These correspond to values for $\log \left(L_{6 \mathrm{~cm}} / L_{60 \mu \mathrm{m}}\right)$ of -4.3 to 1.8 for radio-loud objects and -5.8 to -4.3 for radioquiet objects (assuming a typical optical $-60 \mu \mathrm{m}$ conversion for the BQS quasars - Spinoglio et al 1995). From Figure 4 we see that $\log \left(L_{6 \mathrm{~cm}} / L_{60 \mu \mathrm{m}}\right)$ is -2.6 for 
both 3C 120 and OJ 287. We did not observe 3C 273 at $6 \mathrm{~cm}$ due to scheduling difficulties, but using the $6 \mathrm{~cm}$ flux of $34.9 \mathrm{Jy}$ from Kuehr et al. 1981 yields $\log \left(L_{6 \mathrm{~cm}} / L_{60 \mu \mathrm{m}}\right)=-2.3$, making this the radioloudest object in the $12 \mu \mathrm{m}$ sample. Thus, we see that only $\sim 6-8 \%$ of the $12 \mu \mathrm{m}$ sample is radio-loud (3 of $\sim 50$ observed objects; 4 including Mkn 463 which is borderline, having $\left.\log \left(L_{6 \mathrm{~cm}} / L_{60 \mu m}\right)=-4.3\right)$, which is significantly less than the $15-20 \%$ found for the optically-selected BQS quasars, the difference probably being a function of redshift (and the CfA sample includes no radio-loud objects, with the radioloudest objects of that sample being Mkn 231 with $\left.\log \left(L_{6 \mathrm{~cm}} / L_{60 \mu \mathrm{m}}\right)=-4.5\right)$. Furthermore, there is a bimodal distribution of radio-loudness, in that these three objects exceed all the others by a factor of $\gtrsim 100$ in radio-loudness. Although these objects also have flat slopes and compact radio emission, such properties are also observed in a few other objects e.g., Mkn 231). Therefore, the most clearly distinguishing trait of these objects is their high radio luminosity as compared to that at other wavelengths. In each of the three cases where we see this in our sample, the radio emission is thought to be anisotropic, and beamed preferentially (but not necessarily directly) towards us.

\section{Radio Luminosity Functions}

We have constructed radio luminosity functions (RLFs) at $6 \mathrm{~cm}$ for both the $12 \mu \mathrm{m}$ and CfA samples, for individual Seyfert types and for all Seyferts, in order to determine the true RLF for Seyfert galaxies in the local universe. These RLFs have been derived using the $V / V_{\max }$ method (Schmidt 1968; Schmidt \& Green 1983),

$$
\Phi=\frac{4 \pi}{\Omega f \Delta L} \sum \frac{1}{V_{\max }},
$$

where $V_{\max }$ was individually computed for each galaxy in the sample. We followed the method of E87 for calculating the luminosity function of a sample at a wavelength other than the wavelength at which the sample was defined. We thus use

$$
V_{\text {max }}=\min \left(V_{\text {max }, \text { survey }} V_{\text {max }, \text { radio }}\right),
$$

which represents the maximum volume of space accessible by an object detected at the survey wavelength (mid-IR and optical for the $12 \mu \mathrm{m}$ and CfA sample, respectively) and at radio wavelengths. This is equivalent to deriving the RLF from the IR (or optical) luminosity function and from the bivariate radio-IR (or radio-optical) luminosity distribution function (Elvis et al. 1978; Meurs \& Wilson 1984). We stress, however, that these (and all other) bivariate luminosity functions can only be considered as lower limits to the true space density of Seyferts. This is because the most extreme objects (i.e., those with optical/IR fluxes below the optical/IR survey limit, yet radio fluxes above the radio detection limit) will be excluded, having not been included in the sample in the first place, even though they would have been detected in the radio. We used a $6 \mathrm{~cm}$ flux limit of $0.35 \mathrm{mJy}$, (representing a typical $3 \sigma$ noise level of the $20 \mathrm{~cm}$ maps), a $12 \mu \mathrm{m}$ flux limit of $0.30 \mathrm{Jy}$ (corresponding to the survey limit of the $12 \mu \mathrm{m}$ sample), and an optical flux limit of $6.25 \mathrm{mJy}$ at $4500 \mathrm{~A}$ (corresponding to the magnitude limit of $m_{p g}=14.5$ of the CfA sample - Huchra et al. 1992). The fraction $f$ represents that fraction of the objects in the sample which were observed. [f For bins of width 0.4 in $\log L$ and a RLF proportional to $\mathrm{Mag}^{-1}, \Delta L=1$. The errorbars represent the $90 \%$ confidence interval, based on Poisson statistics, calculated using the equations from Gehrels (1986) which are accurate for even very small numbers of data points.

Figure 6 shows the radio luminosity function for both the $12 \mu \mathrm{m}$ and CfA samples of Seyferts (all types combined). Tables 3 and 4 tabulate the values of the RLF for all Seyferts (and as separated into 1s and 2s) in the $12 \mu \mathrm{m}$ sample and the CfA sample, respectively. We fit each RLF to a single power-law (straight lines), with the results plotted on the graph (in each case the points are weighted by the number of objects they represent, hence the lines look higher than would ones which weigh each point evenly). Both RLFs are fitted well by a power-law (solid line with $r=-0.95$ for the $12 \mu \mathrm{m}$ RLF and the dotted line with $r=-0.91$ for the CfA RLF). The $12 \mu \mathrm{m} \mathrm{RLF} \mathrm{has} \mathrm{a} \mathrm{steeper} \mathrm{slope}$ $(-1.01$, vs. -0.72 for the CfA sample). The integrated RLF for the entire $12 \mu \mathrm{m}$ sample is higher than that for the CfA sample, primarily because it is higher

\footnotetext{
${ }^{5}$ For the $12 \mu \mathrm{m}$ sample, 7 objects were not observed because they were too far south to be reached from the VLA, 3 more were not observed because of scheduling limitations, and 13 because they were not known to be Seyferts at the time of SM89 and were not included in our sample. Thus, $f=(71-23) / 71=0.68$. Similarly, for the CfA sample, only one object was not observed due to scheduling constraints and one was not originally known to be a Seyfert, thus $f=(49-2) / 49=0.96$.
} 
at low luminosities (similar results are obtained for $20 \mathrm{~cm}$ RLFs). This, as well as the flatter slope of the CfA RLF could result from low-luminosity Seyfert 1s being underrepresented in the CfA sample when the weak, broad components of their emission lines are diluted beyond recognition in the more distant objects (Persic et al. 1989; Huchra \& Burg 1992). We have also shown for comparison the RLF from Ulvestad \& Wilson (1989), denoted by x's and a dotted line. The RLF of this distance-limited sample agrees with the others above $\log L=38$, but is slightly lower below this level. It appears much lower at the very lowest luminosities, but these points are less meaningful, since they only represent 1-2 objects per bin. Furthermore, part of the difference is caused by the fact that the Ulvestad \& Wilson RLF is based on fluxes measured in the $\mathrm{A}$ and $\mathrm{A} / \mathrm{B}$ arrays, which represent a smaller area of each galaxy, shifting their RLF to the left as compared to our D-array RLF.

Figure 7 shows the RLFs for individual Seyfert types ( $1 \mathrm{~s}$ and $2 \mathrm{~s}$ ) in the $12 \mu \mathrm{m}$ sample. The RLF of Seyfert 1s extends with a similar power-law slope to very high luminosities, while the Seyfert 2s RLF has a sharp high-luminosity cutoff, reminiscent of the cutoff above the $L_{\star}$ knee in the optical luminosity function of normal galaxies. Over most luminosities $(\log L>37.4)$, where we can accurately measure the RLFs of both Seyfert types, we find the space density of Seyfert $2 \mathrm{~s}$ to be $\sim 2$ times that of Seyfert 1s (i.e., about $1 / 3$ are Seyfert 1s), although Seyfert 1s extend to higher luminosities (similar to the far-infrared luminosity functions calculated in RMS). This has implications for the unified model in that, if the $20 \mathrm{~cm}$ emission is isotropic, then there are $\sim 2$ objects observed to be Seyfert $2 \mathrm{~s}$ for each intrinsically similar Seyfert 1. In the context of this (very simplified) model, with orientation to our line-of-sight being the primary factor distinguishing Seyfert 1s from $2 \mathrm{~s}$, this ratio in space density corresponds to a typical half opening angle of the torus (within which an object would be observed as a Seyfert 1) of $\theta \sim \cos ^{-1}(1-0.33) \sim 48^{\circ}$. We also note that the radio-loud objects in the $12 \mu \mathrm{m}$ sample account for only $0.04 \%$ of the integrated luminosity function at $6 \mathrm{~cm}$, however this is only a lower limit as such objects are the most likely ones to be missed when calculating a bivariate luminosity function.

A similar plot is shown in Figure 8 for individual Seyfert types for the CfA sample. We see here and in Table 4 that the space density of Seyfert 2s in the CfA sample is even less than the Seyfert $1 \mathrm{~s}(\sim 0.8$ times as many Seyfert $2 \mathrm{~s}$ ), probably due to the fact that the CfA sample, being selected at optical wavelengths, is biased against heavily reddened Seyfert $2 \mathrm{~s}$ which have had much of their optical flux reprocessed into the far-infrared. This may also explain why the $60 \mu \mathrm{m}$ luminosity function of the $12 \mu \mathrm{m}$ sample was found to be higher than that of the CfA sample for both Seyfert 1s and Seyfert 2s (RMS93).

\section{Summary and Conclusions}

We have used the VLA in the compact D-array to obtain nearly complete 6 and $20 \mathrm{~cm}$ observations for the mid-IR selected $12 \mu \mathrm{m}$ Seyfert Galaxy sample and the optically-selected CfA Seyfert Galaxy sample. We also have analyzed (from E87) $1.5 \mathrm{~cm}$ OVRO data for the CfA sample. The main results are as follows:

There is no significant difference in the average 6 $20 \mathrm{~cm}$ slopes between Seyfert $1 \mathrm{~s}$ and $2 \mathrm{~s}\left(\alpha_{6 \mathrm{~cm}}^{20} \sim 0.7\right)$, consistent with the standard unified model. There is no systematic trend for either Seyfert type to display upward or downward curvature, but a few Seyfert 1s have particularly flat $6-20$ or $1.5-6 \mathrm{~cm}$ slopes.

We have calculated a simple model in which the spatial distribution of the radio and infrared emission from Seyferts comes from two components: (1) an extended/disk component which has the same ratio of radio - infrared flux and a similar luminosity as normal spirals; and (2) a central component with emits relatively more radio luminosity for a given infrared luminosity. The central component contributes significantly to the radio-IR emission from Seyferts, but is much less dominant in normal spirals.

Calculations based on this model describe the following properties of our data: (1) about half of the galaxies have extended emission at $6 \mathrm{~cm}$, which contributes an average of $\sim 33 \%$ to their total flux; (2) Seyferts are shown to have excess $6 \mathrm{~cm}$ emission relative to non-Seyferts of similar far-IR luminosity, by about a factor of two; and (3) among Seyferts, the $6 \mathrm{~cm}$ and $60 \mu \mathrm{m}$ luminosities are linearly proportional over more than the 3 orders of magnitude spanned by our data.

Three objects in the $12 \mu \mathrm{m}$ sample (and none in the CfA sample) are clearly radio-loud, and have extreme properties as compared to the rest of the sample. These objects are the most luminous, have the strongest radio-IR flux ratios, are compact $(R=1)$, 
and have the flattest spectra $\left(\alpha_{6 \mathrm{~cm}}^{20 \mathrm{~cm}} \sim 0\right)$. Thus, there is a clear distinction between these few radioloud objects and the radio-quiet objects which dominate these samples. The fraction of radio-loud objects is significantly less $(\sim 6 \%)$ than in other, higherredshift samples, such as the BQS quasars.

Radio luminosity functions were derived for both the $12 \mu \mathrm{m}$ and CfA samples. Both samples' RLFs are fitted well by a single power-law. The $12 \mu \mathrm{m}$ RLF is slightly higher, especially at low luminosities. The space density of Seyfert $2 \mathrm{~s}$ in the $12 \mu \mathrm{m}$ sample is about 2 times that of Seyfert 1s over their common range in luminosity, but the RLF of Seyfert 1s extends to higher luminosities. In terms of the standard unified model, this ratio in space density corresponds to a typical (half) opening angle of the torus (within which an object would be observed as a Seyfert 1) of $\theta \sim 48^{\circ}$.

We thank the VLA TAC for providing us with the telescope time during programs AE63 and AE76, and the VLA AOC and OVRO personnel who helped us with the data reduction. This work was supported in part by NASA grant NAG $5-1358$. This research has made use of data obtained through the High Energy Astrophysics Science Archive Research Center Online Service, provided by the NASA-Goddard Space Flight Center.

\section{A. Target Selection and Classification}

\section{A.1. The 12 Micron and CfA Samples}

We chose to define our original sample of galaxies from the IRAS Point Source Catalog, Ver. 2, flux limited at $12 \mu \mathrm{m}$ (Spinoglio \& Malkan 1989; hereafter SM89), since that is the IRAS wavelength which most strongly selects for the hot continua universally produced by active nuclei (whether they are thermal or nonthermal) and is long enough to reject nearly all the flux produced by stars in the host galaxy. This original $12 \mu \mathrm{m}$ sample contains the 390 galaxies above a flux limit of $0.30 \mathrm{Jy}$, with $|b| \geq 25^{\circ}$ (to avoid galactic contamination), as well as $F_{60 \mu m} \geq F_{12 \mu m}$ and/or $F_{100 \mu m} \geq F_{12 \mu m}$ (to select galaxies instead of galactic objects). This sample is not only complete down to a $12 \mu \mathrm{m}$ flux limit, but also with respect to bolometric flux of $2 \times 10^{-10} \mathrm{erg} \mathrm{s}^{-1} \mathrm{~cm}^{-2}$ (RMS). This sample, as reported in SM89, contained 59 galaxies known to harbor Seyfert nuclei. Forty-two of these Seyferts are observable from the VLA and it is for these objects that we have obtained 6 and $20 \mathrm{~cm} \mathrm{D-array} \mathrm{obser-}$ vations. (It is now known that several other objects in the original $12 \mu \mathrm{m}$ sample are Seyferts, but were not identified as such at the time of SM89. These objects are properly identified in the Extended $12 \mu \mathrm{m}$ Sample-RMS93; discussed in Appendix A.3).

We have also observed the Seyfert galaxies in CfA Galaxy sample which is complete down to an optical flux limit of $m_{Z w}=14.5$ (Huchra \& Burg 1992). VLA data were presented in Edelson 1987 for 42 of the 50 CfA-sample Seyferts. We have obtained 6 and $20 \mathrm{~cm}$ observations for 6 CfA Seyferts which were added to the sample after that time, as well as several $2 \mathrm{~cm}$ fluxes to compare to the single-dish $1.5 \mathrm{~cm}$ observations from OVRO in E87 (the $2 \mathrm{~cm}$ fluxes being made at much higher resolution are used only to estimate lower limits to the $1.5 \mathrm{~cm}$ fluxes). Four galaxies were added to the final definition of the CfA sample (Huchra \& Burg 1992; Osterbrock \& Martel 1993) after we began this work and thus we don't have VLA observations for these objects.

\section{A.2. Object Classification}

In both Tables 1 and 2, classification into type 1.0, $1.5,1.8,1.9$, and 2 for all galaxies in the CfA sample (including those objects which overlap with the $12 \mu \mathrm{m}$ sample) is from Osterbrock \& Martel (1993), who compiled a consensus from their own observations and several other works (e.g., Huchra \& Burg 1992 and Dahari \& De Robertis 1988) based on optical spectrophotometry. For most galaxies in the $12 \mu \mathrm{m}$ sample only, detailed classification into Seyfert sub-types is not yet available, and thus we have noted the classification simply as type 1 or 2 , based on references in the literature and popular catalogs (e.g., Hewitt \& Burbidge 1989, 1991; Verón-Cetty \& Verón 1991), as well as on some of our own spectra. (However, a work is in progress-Rush, Malkan, \& Spinoglio 1996 - in which we will examine high signal-to-noise spectrophotometry for all Seyferts in the Extended $12 \mu \mathrm{m}$ sample to determine precisely their Seyfert sub-class.) We note that this may slightly skew those results which focus on differences between Seyfert 1s and $2 \mathrm{~s}$ (e.g., the luminosity functions), as it is likely that a handful of $12 \mu \mathrm{m}$-sample objects which we now consider to be Seyfert 2s are actually Seyfert 1.8-1.9s, and thus should be considered Seyfert 1s when dividing the objects into only two classes. (Even though the spectra of a Seyfert 1.8-1.9 looks more like that 
of a Seyfert 2 than a Seyfert 1, we consider them to be Seyfert 1s when using only two classes. This is because the detection of slight broad wings to the optical emission lines indicates the presence of a directly observable broad-line region, which physically defines a Seyfert 1.8-1.9s as being Seyfert 1s-see, e.g., Goodrich 1989, 1990.)

\section{A.3. The Complete List of 12-Micron-Sample Seyferts}

This paper has studied the radio properties of the CfA Seyfert Galaxy sample and of the original $12 \mu \mathrm{m}$ Seyfert Galaxy sample. For completeness, we here mention the Extended $12 \mu \mathrm{m}$ sample (RMS93) and compare its contents to those of the original $12 \mu \mathrm{m}$ sample.

As mentioned in $\S$ A.1, the original $12 \mu \mathrm{m}$ sample was selected from the IRAS Point Source Catalog, Ver. 2 with a $12 \mu \mathrm{m}$ flux limit of $0.30 \mathrm{Jy}$ (SM89). To probe lower fluxes, we selected candidates for the Extended $12 \mu \mathrm{m}$ sample from the IRAS Faint Source Catalog, Ver. 2 and then defined the sample as those galaxies having SCANPI/ADDSCAN whole-galaxy $12 \mu \mathrm{m}$ fluxes above $0.22 \mathrm{Jy}$. By using the FSC-2, which is complete to a lower flux limit than the PSC2 , the extended sample contains over twice as many (893) galaxies.

The original $12 \mu \mathrm{m}$ sample contained 58 galaxies known to harbor Seyfert nuclei at the time of SM89, and is now known to contain at least 71 Seyferts. Similarly, the Extended $12 \mu \mathrm{m}$ sample includes 122 known Seyferts, and it is likely that several galaxies in this sample have yet to be identified as Seyferts. Such objects are more likely to be Seyfert $1.8 \mathrm{~s}, 1.9 \mathrm{~s}$ and $2 \mathrm{~s}$ which are often harder to identify. They are also likely to be found in those positions in multiwavelength parameter space which are usually occupied by Seyferts.

\section{B. Comparison of Compactness Parameter With Other Samples}

We have checked the accuracy of our measured fluxes by comparing our results to those from the $4.85 \mathrm{GHz}$ northern sky survey of Becker et al. (1991), which has $40^{\prime \prime}$ pixel size and 3'.5 angular resolution. Figure 9 shows a graph of the ratio of our tapered $6 \mathrm{~cm}$ flux to the flux from that catalog versus our $6 \mathrm{~cm}$ compactness parameter, $R$. We find, in general, that both values are near one for the majority of objects (meaning that all three fluxes- $\mathrm{S}_{6 c m, l}, \mathrm{~S}_{6 c m, h}$, and $\mathrm{S}_{4.85 G H z}$ - are roughly equal), while those galaxies with values of $R$ much less than one also have low values of the other ratio, implying that they are simply the most extended. That most values are near 1 further implies that there is little evidence for variability between these observations.

We have also compared our $6 \mathrm{~cm}$ compactness parameter to the $2295 \mathrm{MHz}$ flux density from Roy et al. (1994) in Figure 10 (upper limits to the $2295 \mathrm{MHz}$ flux represent 5 times the rms noise in the fringe-frequency spectrum). We see that the detections are mostly of our most compact objects and the non-detections are spread over all values of $\mathrm{R}$. This is as one would expect if the extended sources are resolved by the $275 \mathrm{~km}$ interferometer and thus are less likely to be detected. In fact, only six objects with $\mathrm{R}<0.85$ were detected at $10 \mathrm{~cm}$ and are individually labeled in Figure 10. Three of these objects (NGC 1068, MKN 841, and TOL 1238-364) are among the $10 \mathrm{~cm}$-brightest objects in this plot and thus one would expect them to be easily detectable. Our data also shows NGC 1365 to be one of the brighter radio sources.

However, we do not see the trend claimed in Roy et al. (1994), that compact radio structures are much more common in Seyfert 2s than in Seyfert 1s. This can be explained by the fact that they note only the combined statistics of two optically selected samples (the CfA sample from E87 and the sample from Norris et al. 1990) and the the $12 \mu \mathrm{m}$ sample. When examined individually, we find that there is no significant difference between the detection rates of Seyfert $2 \mathrm{~s}$ and Seyfert 1s in any one of these samples alone. However, the overall detection rate is different in each of these three samples (about 65\%, 50\%, and 20\% in the $12 \mu \mathrm{m}$, CfA, and Norris et al. samples, respectively). The Norris et al. sample has the lowest detection rate of both Seyfert $1 \mathrm{~s}$ and Seyfert 2s ( 5 of 28 and 2 of 6 , respectively), which is likely due to the fact that it is a higher-redshift, fainter sample. This sample also has the most Seyfert 1s and fewest Seyfert 2s observed of the three samples. Thus, when these different samples are averaged together, the low detection rate of the Norris et al. sample artificially drags down the combined 3-sample detection rate of Seyfert 1s far more than the rate for Seyfert $2 \mathrm{~s}$. 
TABLE 3

RAdio Luminosity FunCtion for the 12-Micron SAMPle

\begin{tabular}{ccccccc}
\hline \hline \multirow{2}{*}{$\begin{array}{c}\log L_{\text {rad }}^{a} \\
\left(\mathrm{ergs} \mathrm{s}^{-1}\right)\end{array}$} & \begin{tabular}{c}
$\log \Phi$ \\
\cline { 2 - 6 }$\left(\mathrm{Mpc}^{-3} \mathrm{M}^{-1}\right)$
\end{tabular} & $N$ & $\begin{array}{c}\log \Phi \\
\left(\mathrm{Mpc}^{-3} \mathrm{M}^{-1}\right)\end{array}$ & $N$ & $\begin{array}{c}\log \Phi \\
\left(\mathrm{Mpc}^{-3} \mathrm{M}^{-1}\right)\end{array}$ & $N$ \\
\hline 36.40 & -3.70 & 1 & $\ldots$ & $\ldots$ & -3.70 & 1 \\
36.80 & -4.08 & 1 & -5.44 & 1 & -4.06 & 2 \\
37.20 & -4.00 & 2 & -3.82 & 3 & -3.60 & 5 \\
37.60 & -5.19 & 1 & -4.21 & 2 & -4.17 & 3 \\
38.00 & -6.22 & 1 & -4.62 & 4 & -4.61 & 5 \\
38.40 & -4.61 & 6 & -4.60 & 6 & -4.30 & 12 \\
38.80 & -5.93 & 5 & -5.56 & 2 & -5.40 & 7 \\
39.20 & -6.85 & 1 & -5.78 & 6 & -5.74 & 7 \\
39.60 & $\ldots$ & $\ldots$ & -6.97 & 1 & -6.97 & 1 \\
40.00 & $\ldots$ & $\ldots$ & -6.26 & 1 & -6.26 & 1 \\
40.40 & $\ldots$ & $\ldots$ & -7.40 & 1 & -7.40 & 1 \\
40.80 & -7.15 & 1 & $\ldots$ & $\ldots$ & -7.15 & 1 \\
41.60 & -6.82 & 1 & $\ldots$ & $\ldots$ & -6.82 & 1 \\
43.20 & -9.47 & 1 & $\ldots$ & $\ldots$ & -9.47 & 1 \\
\hline
\end{tabular}

${ }^{a}$ Central luminosity of a bin 0.4 units wide in $\log L$, which is equivalent to a width of 1 magnitude.

TABLE 4

Radio Luminosity Function for the CFA sample

\begin{tabular}{ccccccc}
\hline \hline \multirow{2}{*}{$\begin{array}{c}\log L_{r a d}^{a} \\
\left(\mathrm{ergs} \mathrm{s}^{-1}\right)\end{array}$} & \begin{tabular}{c}
$\log \Phi$ \\
\cline { 2 - 6 }$\left(\mathrm{Mpc}^{-3} \mathrm{M}^{-1}\right)$
\end{tabular} & $N$ & $\begin{array}{c}\log \Phi \\
\left(\mathrm{Mpc}^{-3} \mathrm{M}^{-1}\right)\end{array}$ & $N$ & $\begin{array}{c}\log \Phi \\
\left(\mathrm{Mpc}^{-3} \mathrm{M}^{-1}\right)\end{array}$ & $N$ \\
\hline 36.00 & -4.08 & 1 & $\ldots$ & $\cdots$ & -4.09 & 1 \\
36.40 & -4.22 & 1 & $\ldots$ & $\ldots$ & -4.23 & 1 \\
36.80 & -4.90 & 1 & $\ldots$ & $\ldots$ & -4.91 & 1 \\
37.20 & -4.33 & 2 & -4.97 & 1 & -4.25 & 3 \\
37.60 & -4.87 & 5 & -4.39 & 5 & -4.25 & 10 \\
38.00 & -4.90 & 2 & $\ldots$ & $\ldots$ & -4.91 & 2 \\
38.40 & -5.03 & 10 & -4.87 & 5 & -4.63 & 15 \\
38.80 & -5.35 & 6 & -5.68 & 1 & -5.18 & 7 \\
39.20 & -5.98 & 2 & -5.73 & 3 & -5.53 & 5 \\
39.60 & $\ldots$ & $\ldots$ & -6.73 & 1 & -6.72 & 1 \\
40.80 & -6.79 & 1 & $\ldots$ & $\ldots$ & -6.80 & 1 \\
\hline
\end{tabular}

${ }^{a}$ Central luminosity of a bin 0.4 units wide in $\log L$, which is equivalent to a width of 1 magnitude. 


\section{REFERENCES}

Antonucci, R.R.J. 1993, ARA\&A 31, 473

Becker, R. H., White, R. L., \& Edwards, A. L. 1991, ApJS 75, 1

Bicay, M.D. \& Helou, G. 1990, ApJ 362, 59

Dahari, O. \& De Robertis, M.M. 1988, ApJS 67, 249

de Bruyn, A.G., \& Wilson, A.S. 1978, A\&A 64, 433

Edelson, R.A. 1987, ApJ 313, 651 (E87)

Elvis, M., Maccacaro, T., Wilson, A.S., Ward, M.J., Penston, M.V., Fosbury, R.A.E., \& Perola, G.C. 1978, MNRAS 183, 129

Gehrels, N. 1986, ApJ 303, 336

Giuricin, G., et al. 1990, ApJS 72, 551

Goodrich, R.W. 1989, ApJ 340, 190

Goodrich, R.W. 1990, ApJ 355, 88

Hewitt, A. \& Burbidge, G. 1989, ApJS 69, 1

Hewitt, A. \& Burbidge, G. 1991, ApJS 75, 297

Huchra, J.P., Geller, M.J., Clemens, C.M., Tokarz, S.P., \& Michel, A. 1992, Bull. C.D.S. 41, 31 (Strasbourg)

Huchra, J. \& Burg, R. 1992, ApJ 393, 90

La Valley, M.P., Isobe, T. \& Feigelson, E.D. 1992, BAAS 24, 839

Kellerman, K.I., Sramek, R., Schmidt, M., Shaffer, D.B., \& Green, R. 1989, AJ, 98, 1195

Kuehr, H., Witzel, A., Pauliny-Toth, I. I. K., \& Nauber, U. 1981, A\&AS 45, 367

Low, F. J., Huchra, J. P., Kleinmann, S. G., \& Cutri, R. M. 1988, ApJ 327, L41

Meurs, E.J.A. \& Wilson, A.S. 1984, A\&A 136, 206

Miller, L., Peacock, J.A., \& Mead, A.R.G. 1990, MNRAS 228, 501

Norris, R.P., Allen, D.A., Sramek, R.A., Kesteven, M.J., \& Troup, E.R. 1990, ApJ 359, 291

Osterbrock, D.E. \& Martel, A. 1993, ApJ 414, 552

Persic, M. et al. 1989, ApJ 344, 125

Roy, A.L., Norris, R.P., Kesteven, M.J., Troup, E.R., \& Reynolds, J.E. 1994, ApJ 432, 496

Rush, B., Malkan, M.A., \& Spinoglio, L. 1993, ApJ 89, 1 (RMS93)

Rush, B., Malkan, M.A., \& Spinoglio, L. 1996, in prep.
Schmidt, M. 1968, ApJ 151, 393

Schmidt, M. \& Green, R.F. 1983, ApJ 269, 352

Spinoglio, L. Malkan, M.A., Rush, B., Carrasco, L., \& Recillas-Cruz, E. 1995, ApJ, in press.

Spinoglio, L. \& Malkan, M.A. 1989, ApJ 342, 83 (SM89)

Verón-Cetty, M.-P. \& Verón, P. 1991, A Catalog of Quasars and Active Nuclei, 5th ed. ESO Scientific Report No. 10, Oct 1991 (Munich: E.S.O.)

Ulvestad, J.S. \& Wilson, A.S. 1984a, ApJ 278, 544

Ulvestad, J.S. \& Wilson, A.S. 1984b, ApJ 285, 439

Ulvestad, J.S., \& Wilson, A.S. 1989, ApJ 343, 659

White, R.L., \& Becker, R.H. 1992, ApJS 79, 331

Wilson, A.S. \& Colbert, E.J.M. 1995, ApJ 438, 62

This 2-column preprint was prepared with the AAS LATEX macros $\mathrm{v} 4.0$. 


\section{FIGURE LEGENDS}

Figure 1 - Radio spectral slope from 6-20 cm versus the $1.5-6 \mathrm{~cm}$ slope. Solid line indicates where $\alpha_{1.5}{ }^{6}=\alpha_{6}{ }^{20}$. The box encloses those points to the bottom left with both slopes steeper than -0.6 . In this and all plots following (unless otherwise specified) filled symbols are Seyfert 1s (including 1.0, 1.5, 1.8, and 1.9) and open symbols are Seyfert 2s. For both Seyfert 1s and 2s: square $=12 \mu \mathrm{m}$ sample only; triangle $=$ CfA sample only; circle $=$ in both the $12 \mu \mathrm{m}$ and CfA samples. On this and all following plots (except the luminosity functions), the single errorbar shown represents a typical intrinsic mesurement uncertainty for the parameters plotted.

Figure 2 - Diagram showing the difference between the $6-20 \mathrm{~cm}$ slope derived from single-dish measurements and the same slope as derived from our VLA data, versus the VLA slopes.

Figure $3-6 \mathrm{~cm}$ luminosity versus $6 \mathrm{~cm}$ compactness parameter. The straight solid line is the best fit to all the data points, and the dashed line is the best fit to all except the 7 most luminous (labeled) points. The curved lines represent models calculated for different values of the extended luminosity at $6 \mathrm{~cm}$ (see text).

Figure 4 - Radio $(6 \mathrm{~cm})$ versus infrared $(60 \mu \mathrm{m})$ monochromatic luminosities. The dotted lines represent various fits to this relation, for this and other data sets, with the slope constrained to 1 . Solid lines represent calculated models (see text for model parameters).

Figure 5-IRAS 25-60 $\mu \mathrm{m}$ slope versus $6 \mathrm{~cm}$ compactness parameter. Horizontal dashed lines represent estimated central and extended values of the IRAS slope. Curves are calculated models: solid curves represent lower values of central $6 \mathrm{~cm}-60 \mu \mathrm{m}$ flux ratios and dotted lines represent higher values.

Figure 6 - Radio Luminosity Function for all objects combined (Seyfert 1s and 2s) in both the $12 \mu \mathrm{m}$ sample (filled circles; offset slightly for clarity) and the CfA sample (open circles), and in Ulvestad \& Wilson (1989; x's). Errorbars represent the 90\% confidence interval, based on Poisson statistics, accurate for very small numbers of data points. Points with no error bars represent just one object in that bin. x's represent the RLF from Ulvestad \& Wilson (1989).

Figure 7 - Radio Luminosity Function for individual Seyfert classes in the $12 \mu \mathrm{m}$ sample. Filled squares are Sy 1 s and open squares are Sy 2s.

Figure 8 - Radio Luminosity Function for individ- ual Seyfert classes in the CfA sample. Filled triangles are Sy 1s and open triangles are Sy 2s.

Figure 9 - The ratio of our tapered $6 \mathrm{~cm}$ flux to the $4.85 \mathrm{GHz}$ flux from Becker et al. (1991) versus our $6 \mathrm{~cm}$ compactness parameter, $\mathrm{R}$, for all objects with detections in each work. The dotted lines represent values of 1 for either ratio.

Figure $10-2295 \mathrm{MHz}$ flux from Roy et al. versus our $6 \mathrm{~cm}$ compactness parameter. Objects detected at $2295 \mathrm{MHz}$ and with $\mathrm{R}<0.85$ are individually labeled. 